\title{
Posterior Acoustic Enhancement in Hepatocellular Carcinoma
}

\author{
Katherine E. Maturen, MD, Ashish P. Wasnik, MD, Janet E. Bailey, MD, Ellen G. Higgins, PA-C, \\ Jonathan M. Rubin, MD, PhD
}

D. Article includes CME test

Received August 10, 2010, from the Department of Radiology, University of Michigan Health System, Ann Arbor, Michigan USA. Revision requested September 15, 2010. Revised manuscript accepted for publication September 29, 2010.

Address correspondence to Katherine E. Maturen, MD, Department of Radiology, B1 D530H, University of Michigan Health System, 1500 E Medical Center Dr, Ann Arbor, MI 48109-5030 USA.

E-mail:kmaturen@umich.edu
Objectives - The purpose of this study was to assess sonographic appearances of hepatocellular carcinoma with particular attention to posterior acoustic effects.

Methods-We performed an Institutional Review Board-approved retrospective review of patients with hepatocellular carcinoma who had undergone sonographically guided procedures in our department between 2001 and 2010. A total of 247 masses thought to represent hepatocellular carcinoma were identified; 27 were excluded because of prior angioembolization (altering the sonographic appearance), alternate histologic diagnoses, and incomplete patient information or imaging. Ultimately, 220 masses in 185 patients ( 138 men and 47 women; average age, 59.1 years) constituted the study population. Preprocedure sonograms were reviewed in consensus by $3 \mathrm{ab}-$ dominal radiologists; the liver echo texture, lesion echogenicity, and posterior acoustic effect were rated and correlated with patient data.

Results - The average mass size was $3.1 \mathrm{~cm}$ (range, $0.7-17 \mathrm{~cm}$ ). In total, $84.1 \%$ of the masses $(n=185)$ arose in abnormally echogenic/attenuating livers; $54.1 \%$ of the masses $(\mathrm{n}=119)$ were predominantly hypoechoic, $23.2 \%(\mathrm{n}=51)$ isoechoic, and $22.7 \%(\mathrm{n}=$ 50) hyperechoic. Target-type morphologic characteristics were noted in 41 masses. Many masses $(52.7 \%[\mathrm{n}=116])$ had no specific posterior acoustic effect, but nearly half $(46.4 \%)$ had either mild $(n=64)$ or marked $(n=38)$ posterior acoustic enhancement. The remaining masses $(0.9 \%[n=2])$ had posterior shadowing. Posterior acoustic enhancement was most common among hyperechoic masses (62\% with posterior acoustic enhancement), target-type masses (63\%), and masses larger than $5 \mathrm{~cm}$ (81.5\%).

Conclusions - Posterior acoustic enhancement is present to some degree in almost half of hepatocellular carcinomas, which may relate to the tissue characteristics of the tumor or the cirrhotic liver itself. Attention to this finding, including scanning without spatial compounding, is recommended during sonographic screening for hepatocellular carcinoma in the growing population of patients with liver disease.

Key Words_cirrhosis; hepatocellular carcinoma; hepatocellular carcinoma screening

$\mathrm{H}$ epatocellular carcinoma is a common and frequently fatal cancer relentlessly increasing in incidence. It is at least the third leading cause of cancer death in the world and the ninth leading cause in the United States ${ }^{1}$; these rates are likely underestimates because the most recent figures from the US Centers for Disease Control and Prevention required histologic diagnosis, whereas the clinical trend is toward acceptance of characteristic imaging as the standard for diagnosis. ${ }^{2,3}$ The incidence of hepatocel- 
lular carcinoma will almost certainly continue to increase in the coming decades, ${ }^{1,4}$ and the importance of imaging for diagnosis and management remains paramount.

Screening protocols for cirrhotic patients at high risk for hepatocellular carcinoma have varied in their particulars but generally include some combination of medical surveillance, serum biomarkers including $\alpha$-fetoprotein, and sonography. ${ }^{5,6}$ The sensitivity of sonography for detection of small lesions is limited, ${ }^{7,8}$ particularly in patients with advanced steatosis or cirrhosis causing increased background parenchymal echogenicity. The sonographic appearance of hepatocellular carcinoma varies with the degree of cellular differentiation and the presence of internal hemorrhage or necrosis, among other factors. ${ }^{9-11}$

Given the large patient population undergoing screening and the potential importance of early detection for these patients, optimization of technique is essential and requires both modern high-resolution sonographic equipment and awareness (by both sonographers and radiologists) of any characteristic imaging features that may improve diagnostic performance in these difficult examinations. Having incidentally noted the presence of posterior acoustic enhancement associated with known hepatocellular carcinoma during a number of sonographically guided interventional procedures, we reviewed our hepatocellular carcinoma database to determine the frequency of this potentially useful finding.

\section{Materials and Methods}

Institutional Review Board approval was obtained for a Health Insurance Portability and Accountability Actcompliant retrospective review of sonograms obtained in conjunction with biopsy or radio frequency ablation procedures performed from September 2001 through March 2010. The patient list was assembled from the crosssectional interventional radiology database using the search term "hepatocellular carcinoma." Images (between 1 and 5) were saved on the radiology archive for each mass, sometimes including cine clips, obtained on a variety of ultrasound machines. Imaging techniques, including the machines and transducers used, varied over the study period, but 3- to 7-MHz transducers are typically used for liver imaging at our institution. Three subspecialist abdominal radiologists (with 4, 5, and 12 years of postresidency experience, respectively) reviewed these images in a consensus panel and tabulated imaging characteristics. Patient records were reviewed to confirm the pathologic diagnosis and establish the presence and type of underlying liver disease, results of other imaging studies, and basic demographics.
A total of 247 masses believed to represent hepatocellular carcinoma in 205 patients were initially identified, and imaging was reviewed. Twenty-seven masses in $22 \mathrm{pa}-$ tients were excluded: 17 masses in 12 patients were in fact metastases from other primary cancers; 5 masses had undergone prior transarterial chemoembolization, altering imaging characteristics; 2 had no images saved in the radiology archive; 2 had no pathologic or clinical information on the record review; and 1 mass was not visible on the imaging review. Two of the above 22 patients had 1 mass excluded and 1 mass included and therefore remained under consideration.

A total of 185 patients thus constituted the study population, including 138 men and 47 women. The average age was 59.1 years (range, 23-89 years). Of the 185 patients, 21 had no known liver disease before or after the diagnosis of hepatocellular carcinoma. The remaining 164 patients had diagnoses of (in decreasing order of frequency): hepatitis $\mathrm{C}$, alcoholic cirrhosis, hepatitis $\mathrm{B}$, cryptogenic cirrhosis, hemochromatosis, primary biliary cirrhosis, $\alpha-1$ antitrypsin deficiency, autoimmune hepatitis, nonalcoholic steatohepatitis, and Budd-Chiari syndrome.

Ultimately, 220 masses remained under consideration. The diagnosis of hepatocellular carcinoma in the index mass was established by biopsy, resection, or pathologist review of the explanted liver for 149 masses (68\%). The index mass size and anatomic site were carefully correlated with biopsy images, surgical reports, and explant review reports to confirm the lesion identity. An additional 30 masses (14\%) had classic imaging findings in the context of a prior tissue diagnosis of hepatocellular carcinoma in another mass in the same patient.

Finally, the diagnosis of hepatocellular carcinoma was established by imaging alone for 41 masses (18\%). This category required an arterially enhancing mass in a cirrhotic liver, as shown by multiphasic computed tomography or magnetic resonance imaging, with relative de-enhancement on delayed imaging 2,3 to be considered presumptive hepatocellular carcinoma by the liver tumor board and physicians performing interventions. All of the patients in this group had documented chronic liver disease from a variety of etiologies. Twenty-nine of these masses were associated with elevated serum $\alpha$-fetoprotein levels; 10 were associated with normal $\alpha$-fetoprotein levels; and laboratory data were not available for 2 masses.

\section{Results}

Readers subjectively rated the echo texture of the background liver parenchyma. The surrounding liver was con- 
sidered normal for only 35 masses (15.9\%), whereas most were somewhat $(61.4 \%[n=135])$ or markedly $(22.7 \%$ $[\mathrm{n}=50])$ echogenic or attenuating. The average lesion size was $3.1 \mathrm{~cm}$ (range, $0.7-17 \mathrm{~cm}$ ); 5 patients had diffuse (nonmeasurable) disease. Most masses were hypoechoic compared with background parenchyma $(54.1 \%[\mathrm{n}=119])$, with smaller numbers of isoechoic $(23.2 \%[\mathrm{n}=51])$ and hyperechoic $(22.7 \%[\mathrm{n}=50])$ masses. Note was also made of the presence of target-type morphologic characteristics (a hypoechoic rim surrounding a mass of variable echogenicity), which were present in 41 masses (Figure 1).

Readers subjectively rated the dominant posterior acoustic effect of the masses in the following 4 categories: (1) definite or marked posterior acoustic enhancement (Figure 2); (2) some or mild posterior acoustic enhancement (Figure 3); (3) no specific effect; and (4) shadowing. Results are shown in Table 1.

Figure 1. Sonogram from a 55-year-old man with hepatitis $C$ and hepatocellular carcinoma. An isoechoic mass (black arrows) with a hypoechoic target-like rim shows mild posterior acoustic enhancement (white arrows).

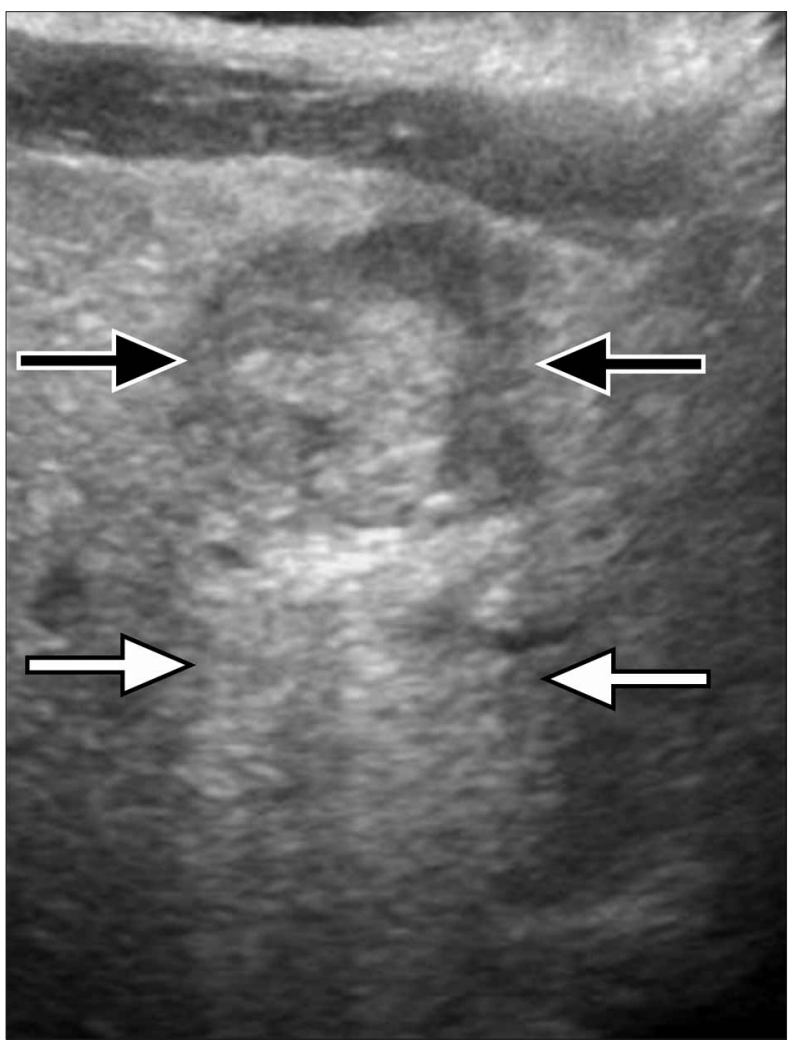

\section{Discussion}

Acoustic enhancement arises posterior to any lesion that attenuates sound less than the surrounding tissue; the intensity of the transmitted ultrasound beam is relatively preserved distal to the lesion. ${ }^{12}$ Posterior acoustic enhancement is most commonly discussed in the context of cystic lesions, which attenuate sound less than any soft tissue structure, but a lesion need not be fluid filled to show enhancement because the difference in transmission is relative. Therefore, we hypothesized that any lesion with a simple internal architecture (or a homogeneous cell population, such as hepatocellular carcinoma) might show posterior acoustic enhancement in a cirrhotic liver. Indeed, we found that posterior acoustic enhancement was present in nearly half of the hepatocellular carcinomas we assessed, although we did not observe any specific relationship with the background parenchymal echogenicity.

Choi and colleagues ${ }^{11}$ observed a similar frequency of posterior acoustic enhancement in a small population of hepatocellular carcinomas smaller than $5 \mathrm{~cm}$. This observation has not been widely incorporated into clinical practice, perhaps because its specificity remains unknown. For example, the typical sonographic profile of hemangioma (hyperechoic with posterior acoustic enhancement)

Figure 2. Sonogram from a 52-year-old man with cryptogenic cirrhosis and hepatocellular carcinoma. A small mildly hypoechoic mass (calipers) shows marked posterior acoustic enhancement (arrows).

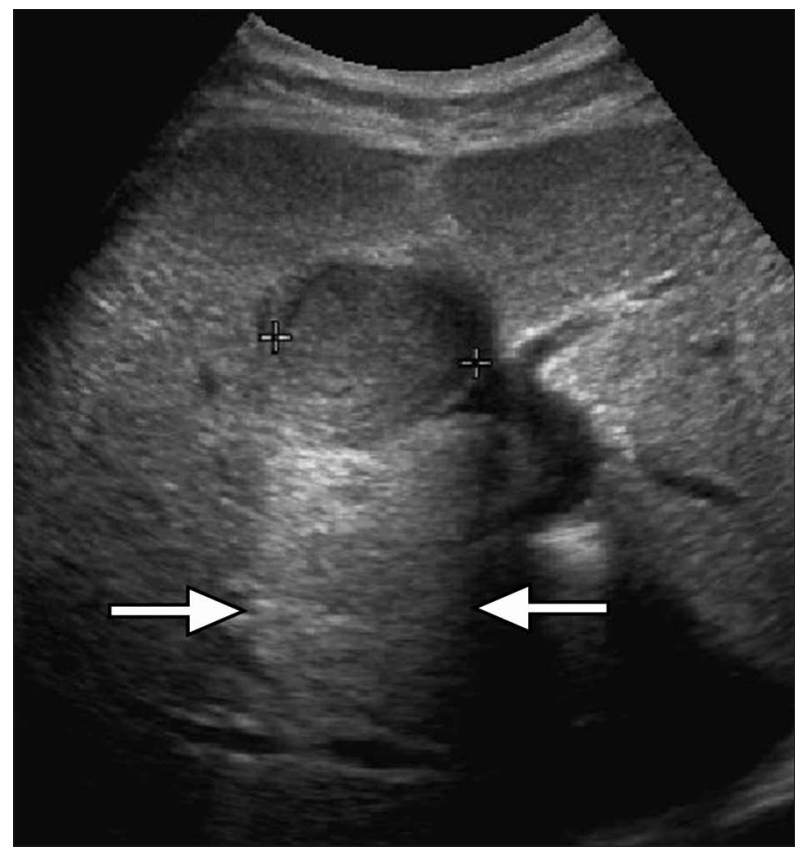


would overlap with 31 hepatocellular carcinomas in our study. The target morphologic characteristics we observed in 41 hepatocellular carcinomas are frequently seen in metastatic disease, ${ }^{13}$ and we have anecdotally observed several metastases with posterior acoustic enhancement, although this observation has not been specifically reported, to our knowledge. However, metastasis and hemangioma are both less common in cirrhotic livers than normal livers, ${ }^{14-16}$ and a high index of suspicion for hepatocellular carcinoma would likely prevail in such patients.

We observed the most acoustic enhancement in association with hyperechoic, target-type, and large lesions. A potential explanation emerges from the tendency of hepatocellular carcinoma to change in sonographic appearance over time. A commonly observed pattern is the small hypoechoic hepatocellular carcinoma gradually becoming hyperechoic, attributed to the development of central necrosis and fibrosis. ${ }^{9,17}$ This observation is in accordance with the histologic identification of a viable "proliferative" cell population at the margin of centrally necrotic and fibrotic tumors, accounting for the hypoechoic rim in many target lesions. ${ }^{18}$ In other words, a welldifferentiated and centrally well-perfused hepatocellular carcinoma is likely to be hypoechoic or isoechoic through-

Figure 3. Sonogram from a 60-year-old man with hepatitis $C$, alcoholic cirrhosis, and hepatocellular carcinoma. A small hypoechoic mass (calipers) shows mild posterior acoustic enhancement (arrows).

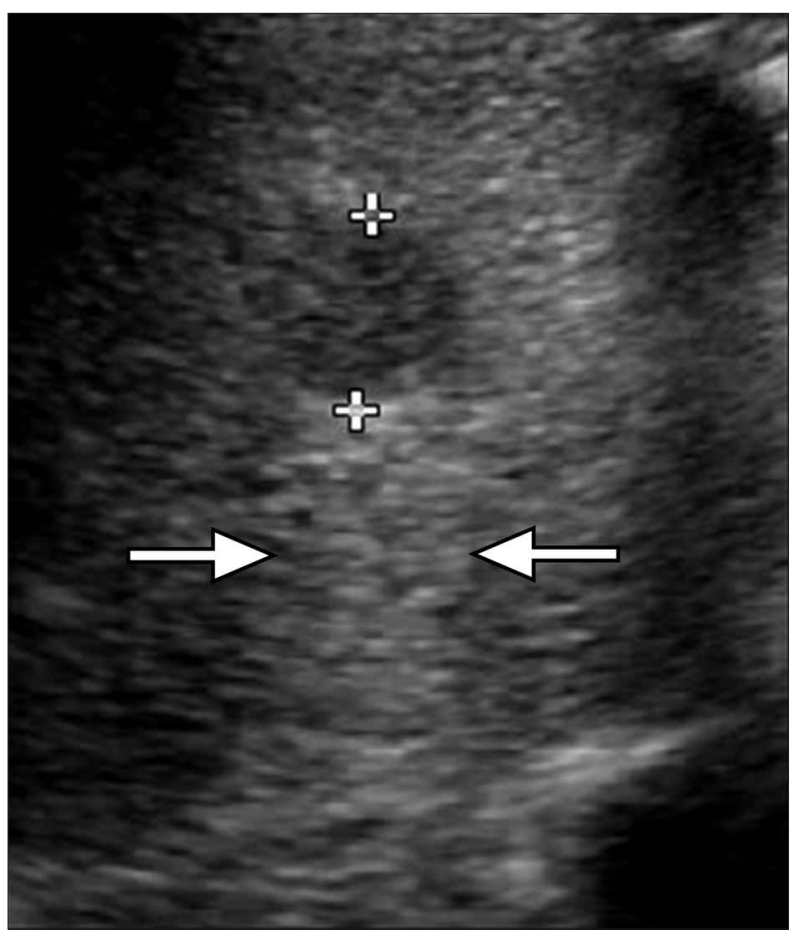

out, whereas a more advanced tumor may become either diffusely hyperechoic or targetlike and larger. The central necrotic component may be more liquefactive than cellular, also decreasing sound attenuation. Thus, posterior acoustic enhancement is more likely to be associated with more advanced cancers, which unfortunately limits the application of this finding in the context of screening for early hepatocellular carcinoma.

Ultrasound scanning techniques are notoriously variable and of clear importance. Spatial compounding is commonly used in sonography to improve the "sharpness" of images by reducing speckle, but its use of multiple nonparallel angles of insonation reduces posterior acoustic effects, including both shadowing and enhancement. ${ }^{19}$ We suggest therefore that screening examinations for hepatocellular carcinoma should include some evaluation without compounding because posterior acoustic enhancement may facilitate detection of a subtle or an isoechoic nodule. In fact, this study may have underestimated the frequency of demonstrable posterior acoustic effects in hepatocellular carcinoma because spatial compounding was routinely left on for interventional procedures.

This study had several limitations. The sonograms reviewed were not diagnostically optimized, intended only to identify masses before intervention. A subset of the cases were not pathologically proven, although all of these arose in the setting of chronic liver disease, most with elevated serum $\alpha$-fetoprotein levels, and all had imaging confirmation in accordance with the current standard of care. ${ }^{2}$ Our pathology reports do not routinely indicate the degree of differentiation or necrosis of hepatocellular carcinoma, so we are unable to provide detailed histologic correlations for the imaging findings. Finally, we are unable to assess the sensitivity or specificity of posterior acoustic enhancement for lesion detection or characterization, respectively, because the population was composed entirely of known hepatocellular carcinomas. Complete evaluation of this imaging sign would require the inclusion of control patients and a prospective approach with a standardized sonographic technique.

In summary, posterior acoustic enhancement is observed in nearly half of hepatocellular carcinomas and may be more frequently associated with advanced cancers. The hepatocellular carcinomas in our study were pleomorphic, with some mimicking benign hemangiomas: echogenic with posterior acoustic enhancement. The sensitivity and specificity of posterior acoustic enhancement for hepatocellular carcinoma remain unknown, but given the increasing hepatocellular carcinoma screening population and the importance of containing medical costs and radi- 
Table 1. Posterior Acoustic Enhancement in Hepatocellular Carcinomas

\begin{tabular}{|c|c|c|}
\hline Category & $\begin{array}{l}\text { Posterior Acoustic } \\
\text { Enhancement (Mild or Marked), \% (n) }\end{array}$ & $\begin{array}{l}\text { No Posterior Acoustic } \\
\text { Enhancement, \% (n) }\end{array}$ \\
\hline \multicolumn{3}{|l|}{ Cumulative } \\
\hline All masses $(n=220)$ & $46.4(102)$ & $53.6(118)^{a}$ \\
\hline \multicolumn{3}{|l|}{ Subset } \\
\hline Target-type masses $(n=41)$ & $63(26)$ & $37(15)$ \\
\hline Hypoechoic masses $(n=119)$ & $46.2(55)$ & $53.8(64)$ \\
\hline Isoechoic masses $(n=51)$ & $31.4(16)$ & $68.6(35)$ \\
\hline Hyperechoic masses $(n=50)$ & $62(31)$ & $38(19)$ \\
\hline Small masses $(<2.5 \mathrm{~cm} ; \mathrm{n}=111)$ & $30.6(34)$ & $69.4(77)$ \\
\hline Medium masses $(2.6-5.0 \mathrm{~cm} ; \mathrm{n}=77)$ & $58.4(45)$ & $41.6(32)$ \\
\hline Large masses $(>5 \mathrm{~cm} ; \mathrm{n}=27)$ & $81.5(22)$ & $18.5(5)$ \\
\hline Highly echogenic background liver $(n=50)$ & $42(21)$ & $58(29)$ \\
\hline Somewhat echogenic background liver $(n=135)$ & $47.5(64)$ & $52.5(71)$ \\
\hline Sonographically normal liver $(n=35)$ & $48.5(17)$ & $51.5(18)$ \\
\hline
\end{tabular}

aTwo masses had posterior acoustic shadowing but were included in the No Posterior Acoustic Enhancement group.

ation doses, we must make the best use of the tools we have. Attention to posterior acoustic effects by sonographers and radiologists, including evaluation without spatial compounding, may contribute to the success of sonographic screening for hepatocellular carcinoma in the cirrhotic liver.

\section{References}

1. Centers for Disease Control and Prevention. Hepatocellular carcinoma-United States, 2001-2006. MMWR Morb Mortal Wkly Rep 2010; 59:517-520.

2. Bruix J, Sherman M. Management of hepatocellular carcinoma. Hepatology 2005; 42:1208-1236.

3. Bruix J, Sherman M, Llovet JM, et al. Clinical management of hepatocellular carcinoma: conclusions of the Barcelona-2000 EASL conference. European Association for the Study of the Liver.J Hepatol 2001; 35:421430.

4. Altekruse SF, McGlynn KA, Reichman ME. Hepatocellular carcinoma incidence, mortality, and survival trends in the United States from 1975 to 2005. J Clin Oncol 2009; 27:1485-1491.

5. Sato T, Tateishi R, Yoshida H, et al. Ultrasound surveillance for early detection of hepatocellular carcinoma among patients with chronic hepatitis C. Hepatol Int 2009; 3:544-550.

6. Noda I, Kitamoto M, Nakahara H, et al. Regular surveillance by imaging for early detection and better prognosis of hepatocellular carcinoma in patients infected with hepatitis C virus. J Gastroenterol 2010; 45:105-112.

7. Dodd GD, Miller WJ, Baron RL, Skolnick ML, Campbell WL. Detection of malignant tumors in end-stage cirrhotic livers: efficacy of sonography as a screening technique. AJR Am J Roentgenol 1992; 159:727-733.

8. Maruyama H, Yoshikawa M, Yokosuka O. Current role of ultrasound for the management of hepatocellular carcinoma. World J Gastroenterol 2008; 14:1710-1719.
9. Sheu JC, Chen DS, Sung JL, et al. Hepatocellular carcinoma: US evolution in the early stage. Radiology 1985; 155:463-467.

10. Lee JY, Choi BI, Han JK, Lee JM, Kim SH. State-of-the-art ultrasonography of hepatocellular carcinoma. Eur J Radiol 2006; 58:177-185.

11. Choi BI, Kim CW, Han MC, et al. Sonographic characteristics of small hepatocellular carcinoma. Gastroint Radiol 1989; 14:255-261.

12. BushbergJ, Seibert J, Leidholdt E, Boone J. The Essential Physics of Medical Imaging. 2nd ed. Philadelphia, PA: Lippincott Williams \& Wilkins; 2002.

13. Wernecke K, Vassallo P, Bick U, Diederich S, Peters PE. The distinction between benign and malignant liver tumors on sonography: value of a hypoechoic halo. AJR Am J Roentgenol 1992; 159:1005-1009.

14. Gervaz P, Pak-art R, Nivatvongs S, Wolff BG, Larson D, Ringel S. Colorectal adenocarcinoma in cirrhotic patients. J Am Coll Surg 2003; 196:874-879.

15. Seymour K, Charnley RM. Evidence that metastasis is less common in cirrhotic than normal liver: a systematic review of post-mortem case-control studies. BrJ Surg 1999; 86:1237-1242.

16. Brancatelli G, Federle MP, Blachar A, Grazioli L. Hemangioma in the cirrhotic liver: diagnosis and natural history. Radiology 2001; 219:69-74.

17. Choi BI, Takayasu K, Han MC. Small hepatocellular carcinomas and associated nodular lesions of the liver: pathology, pathogenesis, and imaging findings. AJR Am J Roentgenol 1993; 160:1177-1187.

18. Wernecke K, Henke L, Vassallo P, et al. Pathologic explanation for hypoechoic halo seen on sonograms of malignant liver tumors: an in vitro correlative study. AJR Am J Roentgenol 1992; 159:1011-1016.

19. Merritt CR. Technology update. Radiol Clin North Am 2001; 39:385397. 